\title{
UNA PUESTA EN VALOR DEL RELIEVE COMO ELEMENTO PATRIMONIAL: GEORUTAS POR EL ALTO CARRIÓN (MONTAÑA PALENTINA)
}

\author{
Ramón PELLITERO ONDICOL
}

Recibido: 09/11/2009

Aceptado: 15/03/2010

RESUMEN: El artículo valora la importancia del relieve como elemento vertebrador del paisaje a la luz de textos y estudios anteriores. Se analizan las distintas iniciativas de protección y explotación del patrimonio geomorfológico, principalmente en el contexto español. Por último se realiza una diagnosis de las potencialidades y debilidades de una comarca en concreto, la Montaña Palentina, se analizan los elementos geomorfológicos sobresalientes presentes en dicha comarca, y se proponen unas rutas geomorfológicas con el fin de poner en valor dicho patrimonio. PALABRAS CLAVE: Patrimonio geomorfológico, georutas, Montaña Palentina, relieve, paisaje.

FRAMING RELIEF AS HERITAGE: GEOROUTES IN THE ALTO CARRIÓN REGION (PALENCIA MOUNTAIN RANGE)

ABSTRACT: This article assesses the significance of landforms as the main support for landscape, based on previous articles and researches. Geoheritage protection and exploitation initiatives are analysed, mainly in Spain. Finally it is performed a diagnosis of potentials and weaknesses the Montaña Palentina county. The outstanding geoheritage features present in the county are assessed, and finally some Geotrails are purposed to value such geoheritage.

KEY WORDS: Geoheritage, geotrails, Palencia Mountain, landforms, landscape.

UNE MISE EN VALEUR DU RELIEF COMME ÉLÉMENT DU PATRIMOINE : GÉOROUTE DE L'ALTO CARRIÓN (MONTAGNE PALENTINA)

RÉSUMÉ: L'article apprécie l'importance du relais comme élément vertebrador du paysage à la lumière de textes et études antérieures. Ils s'analysent les diverses initiatives de protection et exploitation du patrimoine geomorfologique, principalement dans le contexte espagnol. Par dernier se réalise une diagnosis des potentialités et faiblesses d'une région, la Montagne Palentina, s'analysent les éléments geomorfológicos excellents présents en dite région, et se proposent quelques routes geomorfológicas afin de mettre en valeur dite patrimoine.

MOTS-CLÉS: Patrimoine geomorfologique, georoutes, Montagne Palentina, relief, paysage.

UMA VALORIZAÇÃO DO RELEVO COMO ELEMENTO PATRIMONIAL: ROTAS GEOGRÁFICAS PELO ALTO CARRIÓN (MONTANHA PALENTINA)

RESUMO: O artigo valoriza a importância do relevo como elemento vertebrador da paisagem à luz de textos e estudos anteriores. Analisam-se as diferentes iniciativas de protecção e exploração do património geomorfológico, principalmente no contexto espanhol. Por último faz-se um diagnóstico das potencialidades e debilidades de uma comarca, a Montanha Palentina, anali- 
sam-se os elementos geomorfológicos salientes presentes em dita comarca, e propõem-se algumas rotas geomorfológicas para pôr em valor dito património.

PALAVRAS CHAVE: Património geomorfológico, georotas, Montanha Palentina, relevo, paisagem.

\section{LA IMPORTANCIA DEL RELIEVE EN EL ESTUDIO DEL PAISAJE}

El término paisaje surge en Europa durante el Renacimiento, entendido como "porción de terreno visible por un observador", y siendo en primer lugar objeto de interés desde las artes pictóricas (REYNARD, 2009). No será hasta finales del S. XVIII y el S.XIX cuando el paisaje sea observado como "un libro abierto donde se puede descubrir la evolución de la Tierra" (REYNARD, 2009), comenzando a considerarse como objeto de estudio científico, fundamentalmente para la Geografía y la Historia. Ya en el S. XX se desarrollará un estudio humanístico del paisaje, al considerar éste como un archivo donde estudiar procesos naturales y humanos; tales estudios fueron posteriormente criticados, al ignorar cómo los paisajes van mutando con los cambios sociales y económicos del citado siglo. (BERTRAND, 1984). Es durante la década de los 70 cuando la escuela francesa comienza a ver la necesidad de entender y estudiar el paisaje como una realidad ecológica y un producto social cuyo estudio ha de incluir necesariamente ambas dimensiones $\mathrm{y}$, sobre todo, las complejas interrelaciones entre ellas (BERTRAND, 1978). Actualmente el paisaje se entiende como la suma de varias dimensiones, entre las que hay que destacar la biofísica, la cultural, la estética $o$ subjetiva y, como resultado de ésta, la dimensión social (REYNARD, 2009).

El relieve es un factor determinante en la evolución de un paisaje, tanto más cuando nos encontremos en un área rexistática, donde la geomorfogénesis domina la dinámica general de los paisajes (BERTRAND, 1968). Este es el caso de áreas de alta montaña como la Montaña Palentina, donde la variedad litológica, combinadas con la herencia de las glaciaciones cuaternarias y de los procesos geomorfológicos posteriores, generan una amplia geodiversidad. Dicha variedad suele reflejarse en los suelos, la hidrología, los macro y microclimas, en los patrones de acumulación nival y, como consecuencia de todo ello, en una gran diversidad de hábitats y comunidades vegetales (THOMPSON, GORDON y HORSFIELD, 2001). Todo ello hace al relieve el elemento condicionante de los procesos naturales, y por tanto también de los usos y la ocupación humana a lo largo de la historia (GONZÁLEZ y SERRANO, 2009). 


\section{LA PUESTA EN VALOR DEL RELIEVE: EL PATRIMONIO GEOMORFOLÓGICO.}

Los componentes geomorfológicos en el medio ambiente -y por tanto también en el paisaje- pueden dividirse, según RIVAS ET AL. (1997) en dos tipos principales. Por un lado tendríamos los recursos geomorfológicos, constituidos por todos los materiales cuya extracción puede devenir un beneficio económico. Éstos no son renovables, y su consumo provoca un impacto paisajísitico en general alto. Por otro lado tenemos los activos geomorfológicos -las formas de relieve-, recursos cuya puesta en valor no implica extracción directa ni consumo de materiales. Para dicha puesta en valor, estos activos han de ser estudiados y reconocidos. Sin embargo, tal reconocimiento y puesta en valor sólo ha ocurrido recientemente.

Si nos ceñimos al contexto español, ya desde la ley de Parque Nacionales de 1916 se incluye la protección de lugares geomorfológicamente sobresalientes, aunque las razones sean, más allá de las geológicas, de exaltación nacionalista, paisajísticas o cinegéticas (GONZÁLEZ y SERRANO, 2007). La conservación del patrimonio natural en general y del patrimonio geológico en particular se atenúa en las décadas siguientes (BRUSCHI, 2007). Ya en 1989, con la Ley de Espacios Naturales y de la Flora y Fauna Silvestres, se incluyen los aspectos geomorfológicos como objetivo a proteger, aunque de los 469 Espacios Naturales Protegidos declarados en virtud de esta ley en España sólo 79 tengan un componente principalmente geomorfólogico en la motivación de su declaración (BRUSCHI, 2007). Sin embargo, y debido al auge que los estudios sobre Geodiversidad y Patrimonio Geológico han tenido en España en los últimos años, por primera vez estos aspectos han sido nombrados específicamente en la nueva Ley del Patrimonio Natural y la Biodiversidad del año 2007.

El patrimonio geomorfológico constituye un recurso cuya puesta en valor como recurso económico, cultural y educativo es algo relativamente novedoso. La creciente popularidad del ocio al aire libre durante las vacaciones, junto al creciente interés social por el medio ambiente, ha llevado a los turistas a un mayor deseo de contacto con la Naturaleza. Esta atracción, especialmente intensa entre los más jóvenes, ha provocado el aumento de las actividades y deportes de aventura al aire libre (esquí, senderismo, montañismo, escalada, descenso de barrancos, $4 \times 4$, caza y pesca, observación de flora y fauna...). Entre ellas el Geoturismo, entendido como la visita de lugares para disfrutar, apreciar y conocer cómo estos se han formado desde el punto de vista geológico (DOWLING y NEWSOME, 2006), es una actividad en auge. Un recurso óptimo para satisfacer esta demanda es la creación de itinerarios mono o multitemáticos, en los que se 
promueva y explique la variabilidad y complejidad natural de un territorio, explicando sus características geomorfológicas (PELFINI et AL., 2009).

Son muchas las iniciativas de puesta en valor del patrimonio geomorfológico, como la red de Geoparques de la UNESCO, que intenta integrar la protección y el uso sostenible de recursos geológicos con fines educativos, culturales y económicos. Algunos territorios han apostado decididamente por la puesta en valor de recursos geológicos, como la provincia de Teruel, en la que se ha constituido el geoparque del Maestrazgo, y en la que una de las principales atracciones turísticas es el parque temático paleontológico de Dinópolis. El aprovechamiento de paisajes mineros abandonados (las Médulas, Cerro del Hierro en Sevilla o Cabárceno en Cantabria) (BRUSCHI, 2007) es otra de las propuestas más comunes, aunque en el caso de las Médulas y, sobre todo, de Cabárceno el aprovechamiento de su espectacular relieve no haya originado hasta ahora su puesta en valor desde un punto de vista geomorfológico. Por último la apertura de cuevas al turismo es otro ejemplo de aprovechamiento de recursos geológicos, quizá el de mayor tradición y éxito tanto en España como en Castilla y León.

Por otro lado la inclusión de explicaciones sobre Geomorfología en los centros de interpretación de espacios naturales protegidos, o la aparición de gran cantidad de publicaciones geoturísticas -mapas, libros de rutas- (BRUSCHI, 2007) nos hablan de un creciente interés por parte de administraciones regionales y locales en poner en valor el patrimonio geomorfológico como recurso.

\section{LA MONTAÑA PALENTINA, UN ESPACIO DE ABANDONO Y OPORTUNIDAD.}

El Parque Natural de Fuentes Carrionas y Fuente el Cobre - Montaña Palentina se enclava en el extremo N de la provincia de Palencia y, con $783 \mathrm{~km}^{2}$, abarca la mayoría de los espacios montanos de dicha provincia, a excepción de algunas alineaciones montañosas en contacto con la cuenca sedimentaria del Duero, de las sierras situadas en la comarca minera de Barruelo y de los relieves plegados de las loras palentinas. Es un espacio de gran extensión, pudiéndose identificar casi completamente el parque natural con la comarca que tradicionalmente se ha llamado la Montaña Palentina. La Montaña Palentina posee algunas de las características comunes a los medios montanos:

- Unos condicionantes climáticos claros, con temperaturas en general más frías que las áreas circundantes, mayores precipitaciones, y contrastes abruptos entre los valles y las cumbres, o entre las caras septentrionales y meridionales. 
- Un período vegetativo comparativamente más corto que el de las llanuras que la rodean, lo que dificulta los cultivos.

- Unos suelos menos desarrollados que los de la llanura, debido al lavado de materiales ladera abajo y a los citado condicionantes climáticos.

- Una biodiversidad mayor que en la llanura, debida a los citados contrastes climáticos, los gradientes altitudinales y la menor presión antrópica sobre los hábitats.

- Una mayor variedad litológica derivada de una historia geológica más prolongada y variada que en la llanura.

- Una geodiversidad también mayor que en la llanura, derivada de la variedad litológica, de los cambios climáticos cuaternarios y holocenos, y de los contrastes geoecológicos propiciados por el gradiente altitudinal, la variedad de orientaciones y pendientes.

- Un paisaje que, fuera de consideraciones estéticas, es innegablemente más espectacular que el de la llanura.

Influida por dichas ventajas y limitaciones, la Montaña Palentina se configura como un espacio habitado y utilizado históricamente, aunque nunca caracterizado por una excesiva sobrecarga demográfica, cuyas principales potencialidades agrarias se reducen a las formaciones pratenses y los recursos forestales (PLAZA, 1994). Actualmente existen 45 localidades enclavadas dentro del parque, las cuales sólo tenían censados en el año 2008 a 1.491 habitantes (datos INE), lo que arroja una densidad de población de 1,9 habitantes por kilómetro cuadrado. Es por tanto también un espacio fuertemente despoblado, en el que los procesos naturales (colonización vegetal, deslizamientos...) están tomando la iniciativa en la evolución del paisaje. La crisis de las actividades química y minera, pilares industriales de la comarca a lo largo del S. XX (ALARIO, 2002) y de la ganadería extensiva a partir de los años 60 (MARTíNEZ y GARCíA, 1984), han abocado a la comarca al citado despoblamiento generalizado, perdiendo población todos los municipios de la comarca (ALARIO, 2002).

De manera sincrónica a esta crisis ha surgido, dentro de la sociedad actual, la concienciación de que los recursos del medio natural son limitados y sensibles a las actividades antrópicas. En Castilla y León la Ley 8/1991 de Espacios Naturales conllevó la declaración de varios parques naturales, monumentos naturales y paisajes protegidos, declaración cuya finalidad ha sido "la armonización de la protección del patrimonio natural con la promoción del conocimiento y disfrute de los valores naturales y culturales del espacio desde un punto de vista educativo, científico, recreativo y turístico" (artículo 2, Ley 8/1991 de Espacios Natu- 
rales de Castilla y León). Esta intención ha tenido su continuación en distintos programas a escala regional y local (programas LEADER, Programa Parques Naturales de Castilla y León) en los que el turismo de interior, en su variante rural, de caza, de aventura o montaña constituyen una piedra de toque esencial (Plaza y Hortelano, 2005).

\section{GEORUTAS POR LA MONTAÑA PALENTINA: UNA PRO- PUESTA.}

Tanto desde el punto de vista de la protección, como desde el punto de vista de la promoción, el relieve ha jugado un papel secundario en los planes redactados desde las distintas administraciones, siguiendo una tendencia general en todo el panorama conservacionista español (GONZÁLEZ y SERRANO, 2008) e internacional (DIAS y BRILHA, 2004). Ello puede comprobarse en los planes que afectan a la Montaña Palentina, tales como citado el Plan de Ordenación de Recursos Naturales del parque natural en cuestión, o las más recientes Directrices de Ordenación del Territorio de la Montaña Cantábrica Central, en proceso de información pública. Dicho planes ignoran sistemáticamente el hecho de que es el relieve el principal artífice de la belleza y variedad paisajística de la comarca. Como puede observarse en el cuadro siguiente, la Montaña Palentina aglutina un magnífico patrimonio geomorfológico, producto de la variedad de afloramientos rocosos y orientaciones de los cordales, así como del gradiente altitudinal existente. La FIGURA 1, que sigue el modelo propuesto por GORDON et AL. (1998) resume los recursos geomorfológicos de esta comarca.

Figura 1. Resumen de los recursos geomorfológicos presentes en la Montaña Palentina, y valoración subjetiva de su importancia patrimonial (siguiendo a GORDON et AL., 1998).

\begin{tabular}{llc}
\hline Sistema Morfogenético & \multicolumn{1}{c}{ Formas Presentes } & Importancia \\
\hline Formas estructurales & $\begin{array}{l}\text { Mantos de cabalgamiento, fallas, relieve } \\
\text { monoclinal, sinclinales colgados, intrusiones } \\
\text { magmáticas. }\end{array}$ & Nacional \\
\hline Relieve preglaciar & $\begin{array}{l}\text { Valles colgados, laderas regularizadas, gla- } \\
\text { cis, laderas y cimas supraglaciares }\end{array}$ & Regional \\
\hline Formas kársticas & Lapiaces, simas, dolinas, cuevas, surgencias & Local \\
\hline Formas de erosión glaciar & $\begin{array}{l}\text { Circos, artesas y umbrales glaciares, colla- } \\
\text { dos de difluencia, cubetas de sobreexcava- } \\
\text { ción, valles colgados, rocas aborregadas, }\end{array}$ & Regional \\
\hline $\begin{array}{l}\text { Formas de depósito gla- } \\
\text { ciar, yuxtaglaciares o aso- } \\
\text { ciadas a la deglaciación }\end{array}$ & $\begin{array}{l}\text { Morrenas frontales y laterales, mantos de } \\
\text { till, complejos de obturación lateral, terrazas } \\
\text { y abanicos proglaciares, llanuras proglacia- } \\
\text { res, lagunas glaciares, turberas }\end{array}$ & Regional \\
\hline Formas periglaciares & $\begin{array}{l}\text { Glaciares rocosos, lóbulos protalud, "tors", } \\
\text { campos, corrientes y laderas de bloques, ta- }\end{array}$ & Nacional \\
\hline
\end{tabular}




\begin{tabular}{lll}
\hline & $\begin{array}{l}\text { ludes de derrubios, suelos ordenados, cam- } \\
\text { pos de crioturbación, terrazas de crioplana- } \\
\text { ción, derrubios escalonados }\end{array}$ & \\
\hline & $\begin{array}{l}\text { Flujos de derrubios, nichos de nivación, mo- } \\
\text { rrenas de nevero, superficies de abrasión ni- } \\
\text { val, canales de aludes, terracillas, mantos y } \\
\text { lóbulos de solifluxión }\end{array}$ & Nacional \\
\hline $\begin{array}{l}\text { Formas de origen fluvial / } \\
\text { torrencial }\end{array}$ & $\begin{array}{l}\text { Terrazas y abanicos aluviales, cárcavas, va- } \\
\text { lles de incisión fluvial, erosión diferencial. }\end{array}$ & Local \\
\hline $\begin{array}{l}\text { Formas de origen gravita- } \\
\text { cional }\end{array}$ & $\begin{array}{l}\text { Deslizamientos rotacionales, deslizamientos } \\
\text { traslacionales, desprendimientos, formas } \\
\text { "sackung", avalanchas de rocas }\end{array}$ & Regional \\
\hline $\begin{array}{l}\text { Formas de origen antrópi- } \\
\text { co }\end{array}$ & $\begin{array}{l}\text { Terrazas de cultivo, minas y canteras, des- } \\
\text { montes, embalses, karst exhumado }\end{array}$ & Regional \\
\hline
\end{tabular}

Figura 2. El P. N. de Fuentes Carrionas y Fuente el Cobre-Montaña Palentina. Situación de las tres georutas propuestas

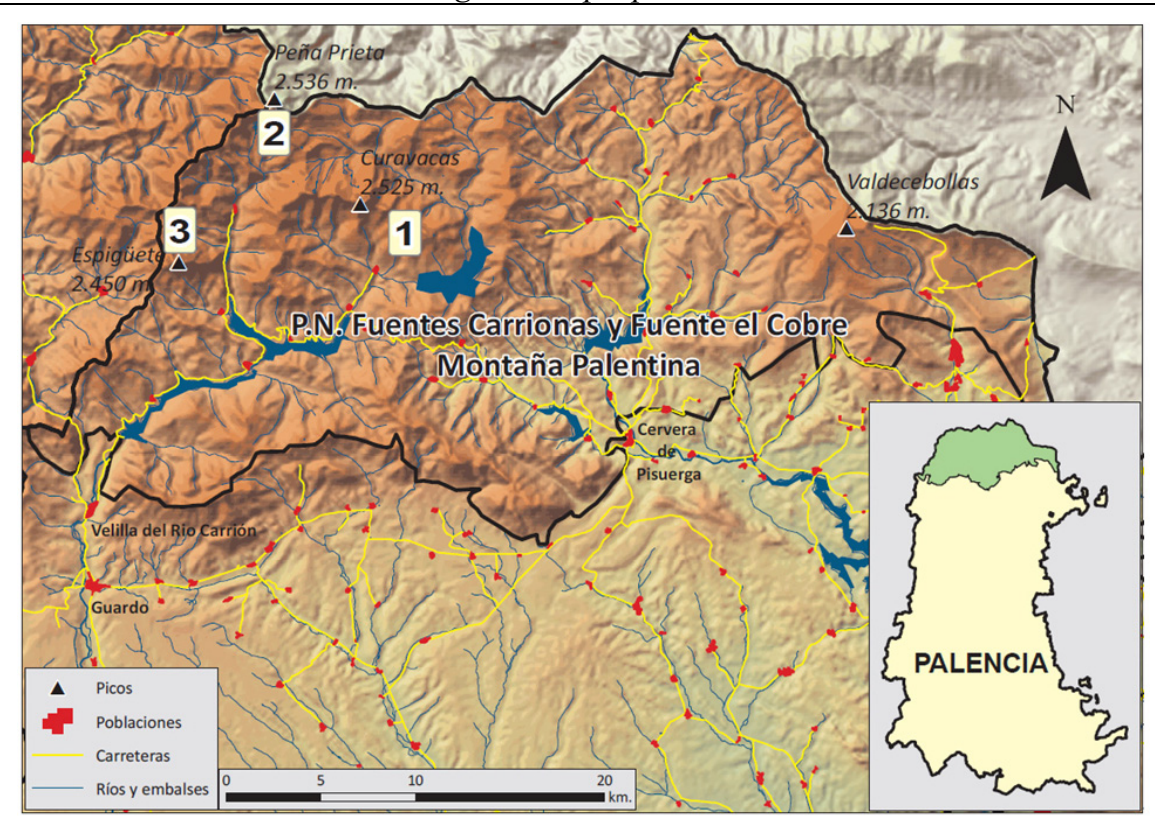

A pesar de la existencia de los citados recursos en la Montaña Palentina, los intentos de aprovechamiento no han pasado de señalizar el acceso a algunos de los lugares más notables, sin promover un verdadero conocimiento del patrimonio geomorfológico, el cual en muchas ocasiones posee un alto valor en el contexto nacional por su originalidad o complejidad. Una excepción a esta regla fueron los itinerarios guiados publicados por ALCALDE CRESPO (1982), los cuáles no tuvieron continuidad en iniciativas de valoración de las formas de relieve 
como recurso patrimonial. Este panorama está cambiando actualmente, y, promovidos desde el entorno académico, desde la administración o desde empresas privadas, existen algunos trabajos que plantean la identificación y evaluación del patrimonio geomorfológico presente en esta comarca (HEREDIA y RODRÍGUEZ, 2001; FERNÁNDEZ-MARTÍNEZ y FUERTES, 2008) o la realización de itinerarios recreativos - didácticos (PELlitero y SERRANo, 2008).

No es objetivo de este artículo el analizar el turismo como fuente de riqueza y sustento social para la comarca, sino que se pretende aportar una alternativa más: la posibilidad de potenciar un turismo que, además de disfrutar del hecho deportivo de pasear por el monte y de la belleza del paisaje, acerque al visitante a la comprensión del mismo desde su elemento vertebrador, el relieve. Las rutas que explican el patrimonio geomorfológico poseen, sobre las rutas que explican o muestran la biodiversidad, la ventaja de que el visitante puede realizarlas de forma autónoma con una guía, y que la mayoría de los recursos geomorfológicos son estáticos, y no cambian estacionalmente (CARCAVILLA, 2007). Además su puesta en explotación no exige ningún tipo de infraestructura, y la forma de realizarlos es a pie, por lo que pueden perfectamente desarrollarse en entornos de gran calidad ambiental, ya que su impacto es mínimo.

Se ha elegido el área de Fuentes Carrionas (FIGURA 2), que aglutina las cumbres de mayor altitud de toda la comarca, y que atrae a una gran cantidad de visitantes, turistas y montañeros, quienes o bien suben a las cumbres más altas o bien recorren los caminos acondicionados para el senderismo, en general sin conocer la génesis de los valles, laderas, paredes, lagos y picos que recorren.

Ruta 1. El Cordal de Lagunillas - Monte las Huelgas hasta el Collado del Hospital.

\begin{tabular}{|c|c|}
\hline Distancia: & 10 kilómetros. \\
\hline Desnivel: & 900 metros. \\
\hline $\begin{array}{l}\text { Duración } \\
\text { aproximada: }\end{array}$ & $6-8$ horas. \\
\hline Dureza: & Media. \\
\hline Dificultad: & $\begin{array}{l}\text { Media - alta. Hasta el Collado de Pando se transita por un cami- } \\
\text { nos y senderos sin dificultades. A partir de aquí no existe senda } \\
\text { marcada, sino que la ruta sigue la cresta cimera. El paso desde el } \\
\text { Monte las Huelgas al Pico del Hospital es algo complicado, ya } \\
\text { que es necesario cruzar una pedrera. Como siempre en alta mon- } \\
\text { taña, la presencia de nieve dificulta la ruta, y obliga al uso de ma- } \\
\text { terial adecuado (piolet y crampones). }\end{array}$ \\
\hline Interés: & Geomorfológico. \\
\hline
\end{tabular}

Esta ruta se encarama al cordal que constituye la prolongación del Curavacas hacia el E. Debido a que la altitud es sensiblemente menor a la del cordal del 
Curavacas (entre 2.000 y 2.200 metros de altitud, frente a los $2.400-2.500$ de éste) y a que el relieve es menos abrupto, puede realizarse cómodamente, con un único paso complicado a la altura del Monte las Huelgas. Con esta ruta el caminante puede hacerse una idea de cómo la presencia de glaciares desde el Último Máximo Glaciar ha propiciado un cambio decisivo en el relieve y el paisaje, ya que durante todo el recorrido camina entre la cara $\mathrm{S}$, donde nunca hubo masas de hielo (PELLITERO, 2008) y los procesos dominantes han sido claramente periglaciares, $\mathrm{y}$ finalmente se asoma a la cara $\mathrm{N}$, donde el relieve glaciar y gravitacional directamente relacionado con la desaparición de las masas de hielo, han labrado un paisaje mucho más abrupto.

Dicho recorrido comienza en la localidad de Vidrieros. La mayor parte del camino discurre sobre un roquedo cuarcítico, en el que vamos a observar un paisaje muy condicionado por la presencia de esta litología, con la existencia de formas asociadas a la presencia de cuarcitas, areniscas o granitos en medios fríos (FRENCH, 2007), sólo cuando nos asomemos a la cara N, desde el cordal de Lagunillas, el paisaje será dominantemente glaciar, y las formas de origen periglaciar pasarán a un segundo plano. Además a partir del Pozo Oscuro pasaremos de las cuarcitas a los conglomerados que forman el macizo del Curavacas.

Figura 3. Imagen en tres dimensiones del recorrido de la georuta 1.

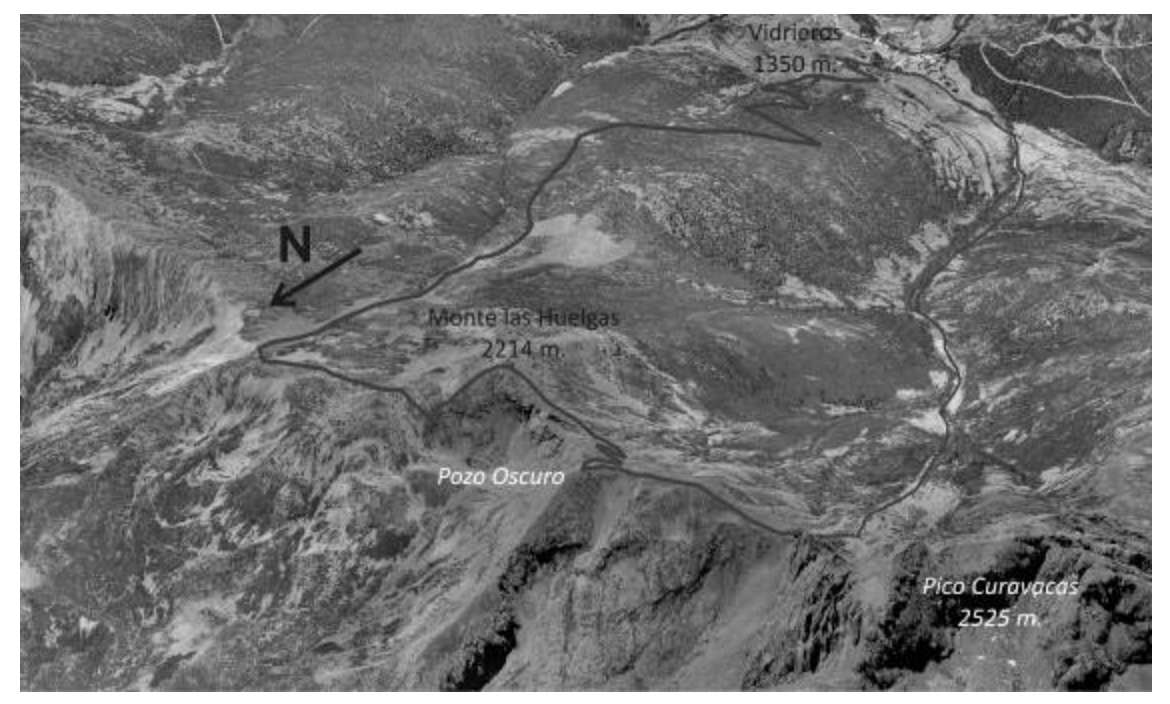

A medida que ascendemos por el camino hacia el collado del Pando, vamos viendo corrientes de bloques cuarcíticos, y laderas regularizadas posiblemente muy antiguas (PELLITERO, 2008). Corrientes, laderas y campos de bloques fue- 
ron originados en un medio periglaciar, con fuertes procesos de hielo - deshielo, gelifluxión y levantamiento de bloques por helada. Estas formas se producen principalmente en cuarcitas porque éstas tienden a disgregarse en bloques decimétricos. Los primeros kilómetros transcurren en un robledal - hayedo, donde también podemos identificar otras especies arbóreas, como olmos, abedules o serbales, entre otros. Pronto el camino asciende por encima del bosque, que aquí desaparece a unos 1.700 metros, y nuestra compañía ahora la componen brezos y piornos, con abedules en los fondos de valle, que no obstante dejan ver la ladera S del Pico Lagunillas, alfombrada con un extenso campo de bloques. En el refugio de Pando, construido en un rellano estructural, podemos tomar el primer descanso, y desde aquí ver el flanco sinclinal del Pico Curavacas, y los voluminosos taludes de derrubios que se han formado a sus pies, y que podremos disfrutar en el último tramo de la ruta. En el refugio dejamos el camino que hemos venido siguiendo y afrontamos el ascenso hasta el Collado de Pando, a 2040 metros de altitud.

Desde el collado podemos ya asomarnos a la cara N, y veremos el Valle de Pineda, 500 metros por debajo del excursionista. Éste es un buen lugar para entender la dinámica glaciar del macizo, en especial cómo y porqué los glaciares se formaron en la cara $\mathrm{N}$ y no en la cara $\mathrm{S}$. Así podemos ver el relieve glaciar tanto del valle del Arroyo del Agua del Manzano como el de Riuplanes, con familias de morrenas laterales, que indican un periodo de estabilidad del glaciar en ese punto. También desde este punto podemos ver dos glaciares rocosos de pequeño tamaño, en ambos valles. Éstos consisten en una masa de hielo y rocas que fluye ladera abajo. Estos glaciares rocosos son buenos indicadores de un ambiente crionival, con temperaturas medias de $-2^{\circ}$, para los que se ha propuesto una edad entre los 14.000 y 10.000 años B.P. (PELLITERO, 2008). Por último el primer valle citado posee una curiosa superficie de erosión nival que, si accedemos a él a finales de primavera o principios de verano, se encontrará cubierta de nieve, lo que puede ejemplificar perfectamente las diferencias ambientales entre la cara $\mathrm{N}$ y la $\mathrm{S}$.

El camino ahora circula por el cordal cimero hasta el Monte las Huelgas, donde se puede ver el Pozo Oscuro, pequeño lago de origen glaciar cerrado por una morrena frontal muy clara. También en la pared opuesta puede verse un $t a-$ lud de derrubios activo, con derrubios escalonados (PELLITERO, 2008). Por toda la cima los bloques levantados, los suelos ordenados heredados y las terracillas son microformas comunes, con un origen mixto periglaciar/nival.

Desde el Monte las Huelgas hasta el Pico del Hospital encontramos el único paso comprometido, por lo que el camino ha de descender por la ladera S para salvar los torreones conglomeráticos de la pared S del circo de Pozo Oscuro. Ello nos obliga a atravesar una incómoda pedrera, aunque sin ningún peligro. 
Una vez llegados al Pico del Hospital podemos ver el lado E del Pozo Oscuro, y en él los sucesivos deslizamientos rotacionales, avalanchas de rocas y deslizamientos sackung existentes en el lugar (FIGURA 4), cuyo origen parece ser paraglaciar, es decir, debido a la descompresión al desaparecer el hielo del glaciar del Valle de Pineda sobre las paredes laterales.

Por último nos acercamos al collado del Hospital y desde él vemos el Hoyo Muerto, una laguna de origen glaciar cerrada por una morrena frontal y flanqueada por un gran talud de derrubios, la Pedrera Pindia. Desde aquí descendemos hacia el SW hasta encontrar la senda que baja del Pico Curavacas, para con ella volver al punto de partida, el pueblo de Vidrieros.

En resumen, los participantes en esta ruta habrían visto una gran variedad de formas de origen glaciar, periglaciar, nival, fluvial y gravitacional. Especialmente habrán comprendido cómo la influencia glaciar ha sido decisiva en los contrastes paisajísticos entre ambas laderas del macizo recorrido.

Figura 4.Vista interpretada hacia el E del Pozo Oscuro desde el Pico del Hospital

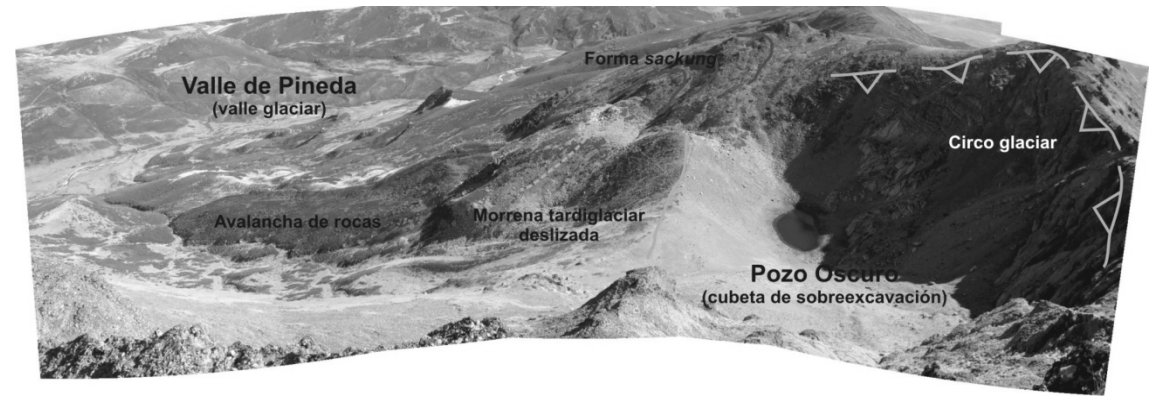

Ruta 2. Ascenso al Mojón Tres Provincias

\begin{tabular}{ll}
\hline Distancia: & 17 kilómetros. \\
\hline Desnivel: & 1100 metros. \\
\hline $\begin{array}{l}\text { Duración } \\
\text { aproximada: }\end{array}$ & $7-9$ horas. \\
\hline Dureza: & Alta. \\
\hline Dificultad: & $\begin{array}{l}\text { Media - baja. Hasta el Pozo de las Lomas se transita por un sen- } \\
\text { dero señalizado. A partir de aquí el sendero está bien marcado } \\
\text { por hitos y por las pisadas de los montañeros. La presencia de } \\
\text { nieve obliga al uso de material adecuado (piolet y crampones). }\end{array}$ \\
\hline Interés: & Geomorfológico. \\
\hline
\end{tabular}

Esta ruta parte de la localidad de Cardaño de Arriba, y en su primera parte sigue el camino señalizado hasta el Pozo de las Lomas. Luego asciende por la 
vertiente $\mathrm{W}$ del Alto del Tio Celestino y crestea por la divisoria entre Fuentes Carrionas y Las Lomas hasta una pradera de altura, donde finalmente el caminante puede, o bien subir al pico Tres Provincias (2.500 metros), divisoria entre las provincias de León, Palencia y Santander, a 20 minutos del citado rellano, o bien acceder a Peña Prieta, máxima altitud del macizo con 2.538 metros. Este último tramo exige 45 minutos adicionales.

A pesar de discurrir por un valle deglaciado, el primer tramo del camino no posee formas de relieve netas, a excepción del relieve diferencial derivado del afloramiento de pizarras y cuarcitas. En el fondo del valle el arroyo de Cardaño ha creado formas de incisión fluvial: pozas y cascadas. A partir de la cascada del Vés, que salva un umbral glaciar, puede seguirse una morrena lateral que el glaciar de las Lomas depositó durante el Máximo Glaciar (FROCHOSO y CASTAÑón, 1996). Después de cruzar el arroyo de Cardaño, y subiendo por la citada morrena, vamos poco a poco alzándonos a un paisaje de cordales, circos $\mathrm{y}$ artesas glaciares en los que frecuentemente las morrenas laterales, fuertemente erosionadas, ofrecen una ruptura de pendiente en las laderas. Así llegamos al entorno del Pozo de las Lomas, lago de sobreexcavación glaciar a 2.057 metros de altitud, flanqueado por las Agujas de Cardaño. Éstas han sido formadas por una sucesión de PROCESOS: en primer lugar la intrusión granodiorítica o "stock" de Peña Prieta (GALLASTEGUI et al., 1990) -fácilmente distinguible de las litologías circundantes por su color grisáceo claro, en contraste con los tonos más oscuros del conglomerado encajante- en segundo lugar la erosión glaciar y, por último, los intensos procesos de crioclastia, que modelaron las agujas hasta darle su forma actual. Al pie de las agujas encontramos espesos canchales taludes de derrubios donde la mayor parte de la fracción gruesa posee tamaños decimétricos-, resultado de la caída de bloques por los citados procesos de crioclastia.

Subimos por una ladera hasta llegar a un campo de bloques supraglaciar, que ocupa la cima del Alto del Tío Celestino. Al llegar a este punto se nos abre hacia el E el espectacular paisaje del Alto Carrión. En primer término tenemos el circo de Fuentes Carrionas, con el lago homónimo, cerrado por un complejo morrénico frontal originado en la última fase glaciar que afectó a este macizo, en la cual los glaciares quedaron limitados a los circos más altos y protegidos. En la ladera $\mathrm{N}$ existe un lóbulo protalud, es decir, un glaciar rocoso incipiente en el que la masa de hielo y rocas apenas fluyó ladera abajo. Todo el circo está cubierto por espesos taludes de derrubios. En un término medio se advierte la forma en U de las artesas glaciares del Valle de Pineda y Coto Redondo, testigos de una difluencia glaciar durante el Máximo Glaciar. Al SE observamos la impresionante cara $\mathrm{N}$ del Curavacas, vaciada también por la erosión glaciar. Por último, al fondo, puede adivinarse el frente de cabalgamiento de la Sierra de 
Híjar - Tres Mares, final de la llamada Montaña Cantábrica e inicio del dominio de pliegues mesozoicos (FIGURA 5).

Figura 5. Imagen en tres dimensiones del recorrido de la georuta 2

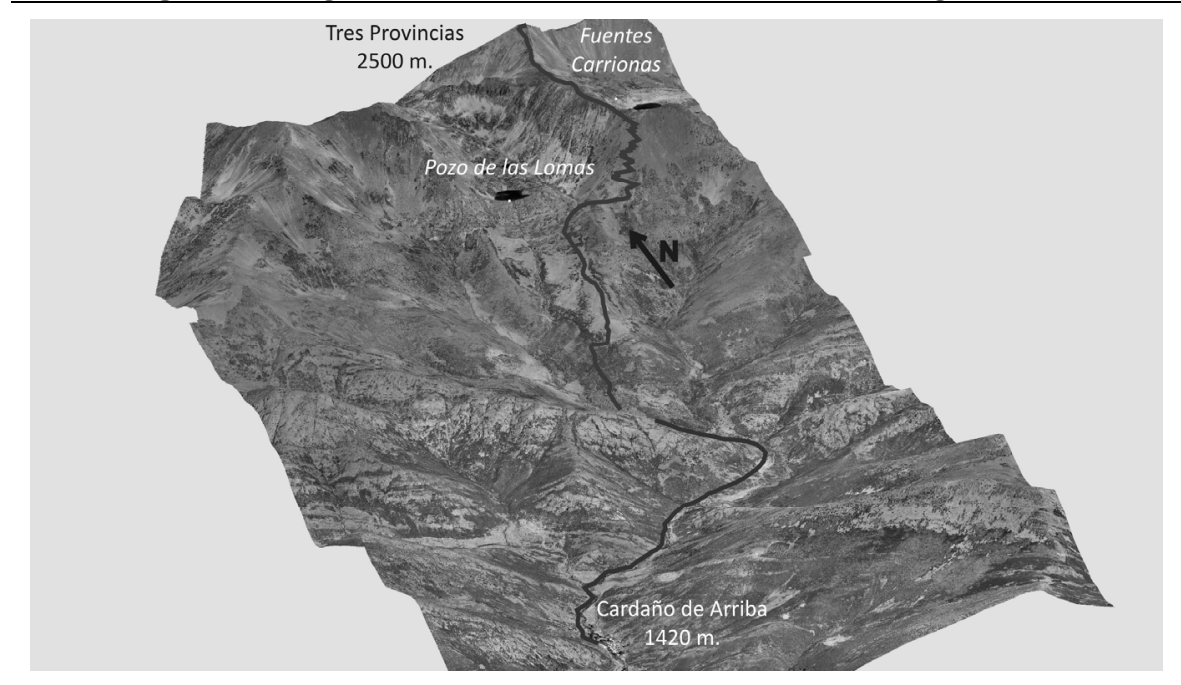

Figura 6. Vista interpretada hacia el E de Fuentes Carrionas desde el camino al Tres Provincias

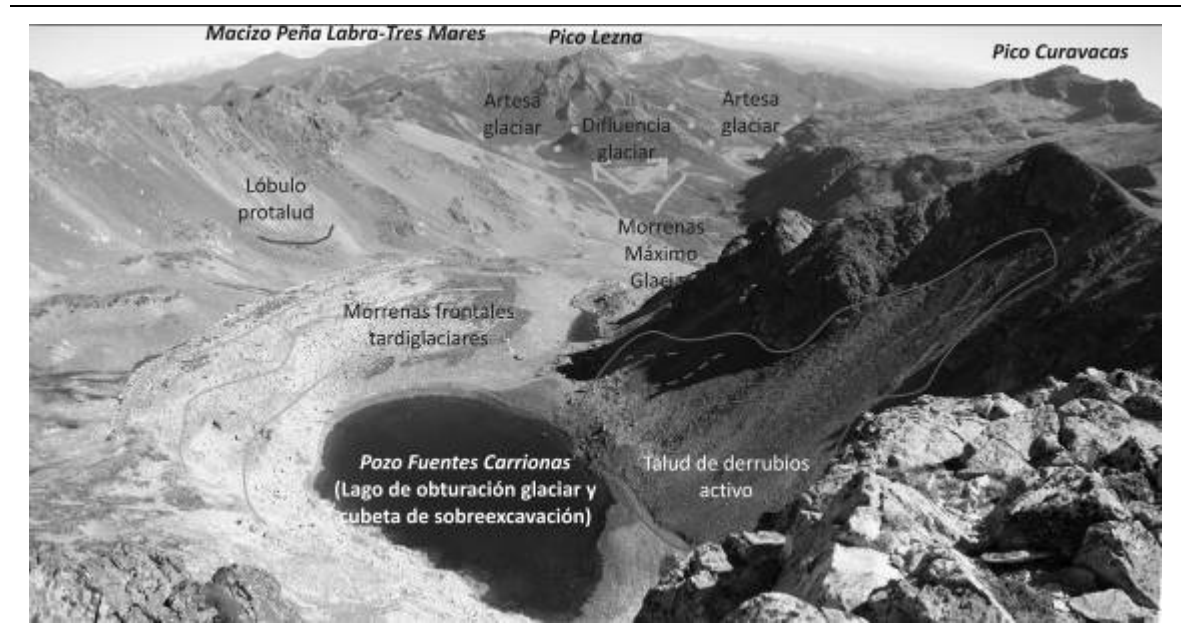

Siguiendo por esta cresta llegamos a una pradera de altura en el límite con la provincia de León. Se trata de una de las praderas más altas de la Cordillera Cantábrica, en la cual las terracillas y los lóbulos de solifluxión, de origen mix- 
to periglaciar y nival, cubren casi toda la superficie. También se han identificado formas exclusivamente periglaciares, como los círculos de piedras, formas relictas que nos hablan de la existencia de suelos permanentemente helados en el pasado (FERNÁNDEZ-MARTÍNEZ y FUERTES, 2008). Desde aquí subimos al pico Tres Provincias, desde el cual vemos al SE el citado valle de Fuentes Carrionas, al NE el circo de los Altares, con el Pozo de los Altares - cubeta de sobreexcavación glaciar- al pie de un talud de derrubios con lóbulos de gelifluxión, y al W el Hoyo Empedrado, un circo glaciar cubierto de clastos granodioríticos procedentes de la ruptura por hielo - deshielo de las paredes del mismo, en el que pueden apreciarse un glaciar rocoso y una morrena de nevero. La vuelta ha de hacerse por el mismo camino, ya que cualquier otra opción alargará y dificultará la ruta.

\begin{tabular}{ll}
\hline & \multicolumn{1}{c}{ Ruta 3. La cara N del Espigüete } \\
\hline Distancia: & 9 kilómetros. \\
\hline $\begin{array}{l}\text { Desnivel: } \\
\text { aproxión }\end{array}$ & 700 metros. \\
\hline Dureza: & Media. \\
\hline Dificultad: & $\begin{array}{l}\text { Baja hasta la sima del Anillo, media-alta a partir de este punto, } \\
\text { con obligación de transitar fuera de caminos o senderos, e incluso } \\
\text { realizar pequeñas trepadas. En todo caso es muy difícil perderse } \\
\text { debido a que siempre circulamos por el mismo valle. Esta ruta no } \\
\text { debería realizarse con nieve, ya que ésta puede ocultar la gran } \\
\text { cantidad de simas existentes en la cara N del Espigüete. }\end{array}$ \\
\hline Interés: & Geomorfológico. \\
\hline
\end{tabular}

Esta ruta aprovecha en su primer tramo el camino señalizado hasta la Cascada de Mazobre desde el aparcamiento de Pino Llano. En ella se circula por el valle de Mazobre, con la vertical cara $\mathrm{N}$ del Espigüete omnipresente a nuestra izquierda. Dicho valle ha sido labrado en el contacto entre las calizas de montaña al S, las cuales forman aquí la característica cima triangular del Espigüete, y al $\mathrm{N}$ formaciones silíceas carboníferas (pizarras y conglomerados), lo cual introduce un claro contraste litológico y cromático, así como de comportamiento de la red hídrica, la cual tiende a circular de manera subterránea en todo el macizo calizo del Espigüete. El valle de Mazobre es un valle glaciar labrado por lenguas de hielo provenientes en su mayoría de la cara $\mathrm{N}$ del Espigüete. Dicha herencia glaciar puede comprobarse en los depósitos de till (material transportado por el glaciar) adosados a la ladera, los cuáles han sido cortados por las obras de adecuación del camino a unos 200 metros de su inicio. Ello ha provocado continuos pequeños deslizamientos y desprendimientos, en especial de bloques, obligando a la posterior construcción de un muro de contención. El 
camino cruza un canal de aludes, en el que pueden verse restos de árboles dislocados por la fuerza de la nieve, dando la posibilidad de explicar este fenómeno tan común en la alta montaña.

Figura 7. Vista hacia el $S$ de la cara $N$ del Espigüete, con la georuta 3 señalizada

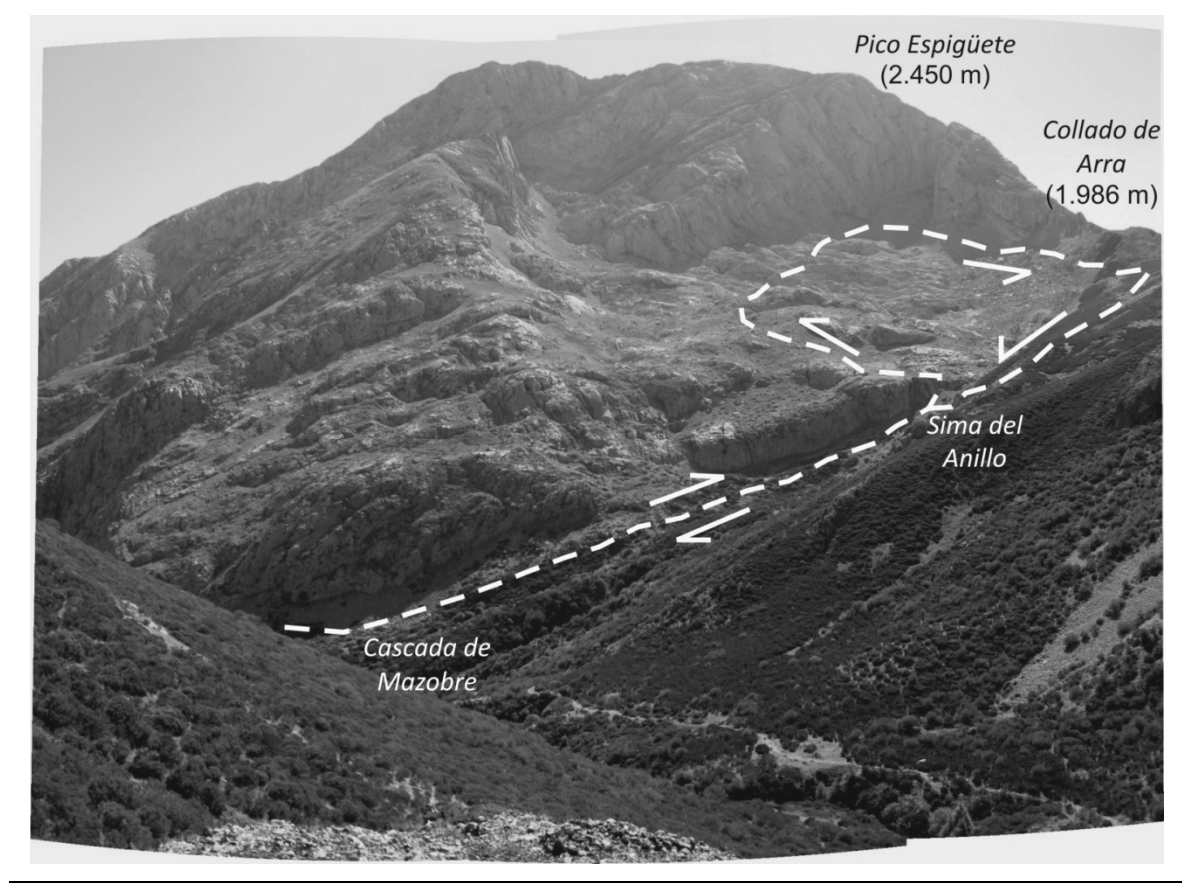

Poco antes de llegar a la Cascada de Mazobre, el camino propuesto se desvía hacia el SW para seguir la pared del Espigüete, circulando por un canal fluvial normalmente seco por la infiltración generalizada del agua en el complejo kárstico que más adelante se revela. Después de una subida algo molesta por la vegetación llegamos a la llamada Sima del Anillo, conducto kárstico de 790 metros de recorrido y 304 metros de profundidad que, formado en el contacto entre las calizas de montaña y las pizarras, recibe todas las aguas de las áreas circundantes.

Desde este lugar se abandona el camino que sube hasta el collado de Arra y vamos a recorrer el Circo $\mathrm{N}$ del Espigüete, que se resume en una superficie pulida por la erosión glaciar y lacerada por la disolución kárstica, por lo que podemos encontrar abundancia de lapiaces de acanaladuras, dolinas, simas, junto con morrenas frontales y laterales, éstas menos visibles. También, a nivel de detalle en el centro del circo, una falla pone en contacto un pequeño afloramien- 
to de caliza oscura con venas de calcita con la caliza de montaña, creándose un contraste bastante visible.

Seguiremos subiendo en dirección SW hasta alcanzar la zona más umbría, donde normalmente se refugian los rebecos, y donde la morfología kárstica continua siendo muy marcada, con abundantes simas y lapiaces de tipo karren. Ésta es una zona de gran actividad geomorfológica, como lo atestiguan las morrenas de nevero y los taludes de derrubios activos. Al W del circo la presencia de un nevero semipermanente ha dejado una clara marca en la pared caliza, análoga a las marcas dejadas por el agua embalsada en las rocas de las orillas de los embalses. Más abajo encontramos un gran desprendimiento que cubre toda la mitad W del circo. La existencia de unas tenues marcas de flujo, sólo visibles en fotografía aérea, nos puede indicar que dicho desprendimiento llegó a funcionar como glaciar rocoso, forma muy poco frecuente en calizas (FIGURA 8).

Figura 8. Vista interpretada hacia el $S$ de la cara $N$ del Espigüete

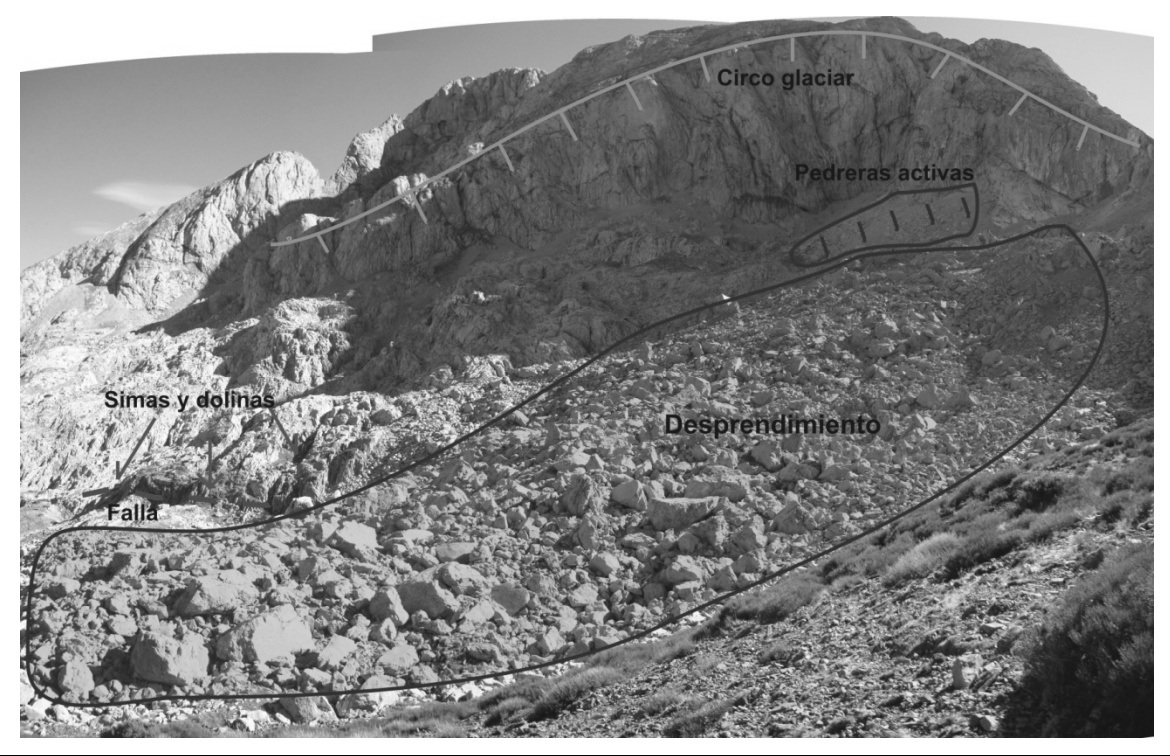

Desde este punto el excursionista puede acercarse al collado de Arra a 1.986 metros de altitud. Dicho collado ha sido usado secularmente por los habitantes de Valverde de la Sierra para acceder (durante la noche) al citado nevero permanente y recoger nieve, que se vendía posteriormente en León, Valladolid y Palencia. Desde este collado también se ve el complejo morrénico frontal del pequeño glaciar que se ubicó en la cara W del Espigüete. 
La vuelta se realizará por la ladera W de la Hoya de Martín Vaquero hasta la sima del Anillo, y desde aquí vuelta hasta la Cascada de Mazobre. Esta cascada (en realidad un grupo de hasta tres cascadas) tiene un origen mixto glaciar-estructural, al cruzar el Arroyo Mazobre capas perpendiculares de conglomerados de la Fm. Curavacas, a las que se adaptó el glaciar del Espigüete, el cual modeló una artesa glaciar que dejó colgado el valle tributario de Mazobres.

\section{CONCLUSIONES}

Las tres georutas propuestas nos permiten ver formas pertenecientes a todos los sistemas morfogenéticos responsables del relieve del alto Carrión (FIGURA 9). La consecución de cualquiera de estas rutas, bastante conocidas entre los aficionados al senderismo y montañismo, permitirán al visitante:

1. Realizar una actividad más completa que la meramente deportiva o admirativa del paisaje.

2. Comprender el porqué del relieve que le rodea.

3. Valorar el patrimonio geomorfológico como parte importante del patrimonio natural.

Todo ello con un impacto sobre el medio ambiente que, de no acometer ninguna señalización o acondicionamiento de las rutas -obra que desaconsejamos, ya que en su mayor parte el recorrido está ya señalizado, y donde no lo está, el paisaje, entendido como suma de sensaciones, pierde su carácter agreste y salvaje-, es mínimo, ya que se limita a una estancia limitada en parajes de alta calidad ambiental.

Figura 9. Sistemas morfogenéticos representados en las distintas rutas geomorfológicas propuestas

\begin{tabular}{lccc}
\hline Sistemas morfogenéticos & ruta 1 & ruta 2 & ruta 3 \\
\hline Formas estructurales & $\checkmark$ & $\checkmark$ & $\checkmark$ \\
\hline Relieve preglaciar & $\checkmark$ & $\checkmark$ & \\
\hline Formas kársticas & & & $\checkmark$ \\
\hline Formas de erosión glaciar & $\checkmark$ & $\checkmark$ & $\checkmark$ \\
\hline $\begin{array}{l}\text { Formas de depósito glaciar, yuxtaglaciares o asociadas } \\
\text { a la deglaciación }\end{array}$ & $\checkmark$ & $\checkmark$ & $\checkmark$ \\
\hline Formas periglaciares & $\checkmark$ & $\checkmark$ & $\checkmark$ \\
\hline Formas nivales & $\checkmark$ & $\checkmark$ & $\checkmark$ \\
\hline Formas de origen fluvial / torrencial & $\checkmark$ & $\checkmark$ & $\checkmark$ \\
\hline Formas de origen gravitacional & $\checkmark$ & & $\checkmark$ \\
\hline Formas de origen antrópico & $\checkmark$ & & $\checkmark$ \\
\hline
\end{tabular}


El relieve, las formas y depósitos que configuran el armazón del paisaje, es un recurso cuya puesta en valor es relativamente reciente en el contexto científico y de gestión de espacios naturales. La Montaña Palentina es un área eminentemente montañosa en una situación de falta de alternativas económicas y crisis demográfica. Sin embargo, las nuevas tendencias de protección y disfrute del medio natural han puesto en valor espacios montanos en los que el patrimonio natural es más valioso que en áreas de llanura. Dentro de dicho patrimonio natural la Montaña Palentina goza de la existencia de un variado patrimonio geomorfológico, frecuentemente desconocido o poco explotado. Las rutas geomorfológicas son un óptimo vehículo para su aprovechamiento, ya que a la vez atraen visitantes, ponen en conocimiento dicho patrimonio en un entorno espectacular desde el punto de vista estético y provocan un impacto mínimo en el medio.

\section{AGRADECIMIENTOS:}

Para la realización de este artículo el autor ha contado con una beca del Programa de Formación de Profesorado Universitario (FPU) del Ministerio de Educación.

\section{BIBLIOGRAFÍA}

ALARIO, M. (2002): «La Montaña Palentina: entre la crisis y la multifuncionalidad». Medio Ambiente en Castilla y León, 5, pp. 19 - 35.

AlCALDE, G. (1982): La montaña palentina. Tomo IV; Fuentes Carrionas y la Peña. Diputación Provincial de Palencia.

BERTRAND, G. (1968): «Paysage et géographie physique globale. Esquise méthodologique». Revue Géographique des Pyrenées et du Sud-Ouest, 39, fas. 2, pp. 249272.

BERTRAND, G. (1978): «Le paysage entre la Nature et la Societé». Revue Géographique des Pyrenées et du Sud - Ouest, 49, fas. 2, pp. 239-258.

BERTRAND, G. (1984): «Les geógraphes français et leurs paysages». Annales de Géographie, 516, pp. 218-229.

BRUSCHI, V. M. (2007): Desarrollo de una metodología para la caracterización, evaluación y gestión de los recursos de la geodiversidad. Tesis doctoral. Universidad de Cantabria.

CARCAVILLA, L. (2007): «La divulgación de la Geología en espacio protegidos: las georutas del Parque Natural del Alto Tajo (Guadalajara)». Enseñanzas de las Ciencias de la Tierra, 15.1, pp. 65-76.

DIAS, G. y Brilha, J.B. (2004): «Raising public awareness of geological heritage: a set of initiatives». En Natural and cultural landscapes: the geological foundation. Parkes, M. A. (Ed.) Royal Irish Academy, Dublin. pp. 235-238.

Dowling, R. y Newsome, D. (2006): Geotourism, sustainability, impacts and management. Oxford, Elsevier. 
FERnÁNDEZ-MARTÍNEZ, E. y FUERTES, I. (Coord.) (2008): Lugares de Interés Geológico. Palencia. Valladolid, Consejería de Medio Ambiente de Castilla y León. CDROM.

FRENCH, H.M. (2007): The Periglacial Environment. 3a Edición. Chichester, Wiley \& Sons.

Frochoso, M. y CASTAÑón, J.C. (1996): «El relieve heredado de la glaciación cuaternaria en el macizo de Peña Prieta (Cordillera Cantábrica)». Polígonos. Revista de Geografia, 6, pp. 25-36.

Gallastegui, G.; Heredia, N.; RodRÍGUEZ, L. R.; CUeSTA, A. (1990): «El stock granítico de Peña Prieta en el contexto del magmatismo de la Unidad del Pisuerga - Carrión (Zona Cantábrica, $\mathrm{N}$ de España)». Cuadernos del Laboratorio Geológico de Laxe, 15, pp. 203-217.

GonzÁlez, J. J. y SERRANO, E. (2007): Cultura y Naturaleza en la Montaña Cantábrica. Universidad de Cantabria.

GonZÁlez, J. J. y SERRANO, E. (2008): «La valoración del Patrimonio Geomorfológico en espacios naturales protegidos. Su aplicación al Parque Nacional de los Picos de Europa». Boletín de la AGE, 47, pp. 175-194.

GONZÁlEZ, J. J. y SERRANO, E. (2009): Geomorfología del Macizo Oriental del Parque Nacional de los Picos de Europa. Naturaleza y Parque Nacionales, serie técnica. Madrid, Ministerio de Medio Ambiente.

Gordon, J. E.; Thompson, B. A.; Haynes, V. M.; Brazier, V. y Mcdonald, R. (1998): «Environmental sensivity and conservation management in the Cairngorm mountains, Scotland». Ambio, 27. ${ }^{\circ} 4$, pp. 335-344.

HEREDIA, N. y RodRÍGUEZ, R. (2001): «El patrimonio geológico de la provincia de Palencia». En Patrimonio Geológico de Castilla y León (R. Nuche, Ed.). Madrid, ENRESA, pp. 190-223.

MARTÍNEZ, S. y GARCÍA, M. A. (1984): «Posibilidades actuales de la ganadería extensiva en zonas de montaña». Actas de las Jornadas de estudios sobre la montaña. León, Urz, pp. 57-86.

Pelfini, M.; Brandolini, P.; CARTON, A. y PicCAZZO, M. (2009): «Geotourist trails: a geomorphological risk - impact analysis». En Geomorphosites (E. REYNARD, P. Coratza, y G. Regolini-Bissig, Eds.). Munich, Verlag Dr. Friedrich Pfeil, pp. 131-144.

PELlitero, R. (2008): El Macizo del Curavacas: mapa geomorfológico e interpretación morfoestratigráfica, geoecológica y paleoclimática. Trabajo de investigación tutelado. Universidad de Valladolid.

Pellitero, R. y Serrano, E. (2008): «La Montaña Palentina: el macizo de Valdecebollas y los valles de Brañosera y Redondos». En De Castilla al Mar: la naturaleza del paisaje en la Montaña Cantábrica. (P. Ruiz, E. SERrano, M.A. Poblete y RUIZ, J., Eds.). Universidad de Valladolid - Universidad de Oviedo, pp. 37-68.

PLAZA, J. I. (1994): «Actividad agraria, valores ambientales y desarrollo rural en espacio natural protegido de «Fuentes Carrionas Fuentelcobre» (Montaña Palentina)». Actas del VII Coloquio de Geografía Rural: comunicaciones. Universidad de Córdoba, pp. 430-434.

PlazA, J. I. y Hortelano, L. A. (2005): «Propuestas institucionales para fomentar el turismo en espacios naturales protegidos: Valoración del "Programa Parques Natura- 
les en Castilla y León” y su incidencia en la Montaña Palentina». Papeles de la Institución Tello Téllez de Meneses, 76, pp. 477-498.

REYNARD, E. (2009): «Geomorphosites and landscapes». En Geomorphosites (E. Reynard, P. Coratza, y G. Regolini-Bissig, Eds.). Munich, Verlag Dr. Friedrich Pfeil, pp. 21-34.

RIVAS, V.; RiX, K.; FrancÉs, E.; CEndero, A. y BRUnSDEN, D. (1997): «Geomorphological indicators for environmental impact assessment: consumable and nonconsumable geomorphological resources». Geomorphology, 18, pp. 169-182.

THOMPSON, B.A.; GORDON, J.E. y HorSFIELD, D. (2001): «Mountain landscapes in Scotland: are these natural, artefacts, or complex relics?» En Earth science and the Natural Heritage: interactions and integrated management. Edimburg, Scotish Natural Heritage, pp. 105-119.

\section{FUENTES CONSULTADAS}

Instituto Nacional de Estadística: Base de datos Nomenclátor.

www.ine.es/nomen2/index.do

Ley 8/1991 de Espacios Naturales de Castilla y León.

Plan de Ordenación de Recursos Naturales del P.N. Fuentes Carrionas y Fuente el Cobre-Montaña Palentina. (1995). Consejería de Medio Ambiente y Ordenación del Territorio de la Junta de Castilla y León. 